\title{
DETECCIÓN DEL INICIO DE REACTIVACIÓN VOLCÁNICA EN LA ISLA DE LA PALMA, ISLAS CANARIAS, Y ESTUDIO DE SU EVOLUCIÓN TEMPORAL
}

\author{
DETECTION OF THE VOLCANIC UNREST ONSET ON LA PALMA ISLAND, CANARY ISLANDS, AND STUDY OF \\ ITS TIME EVOLUTION

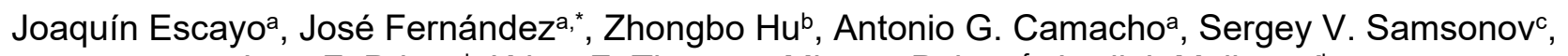 \\ Juan F. Prieto ${ }^{d}$, Kristy F. Tiampo ${ }^{e}$, Mimmo Palanof, Jordi J. Mallorquí ${ }^{b}$ \\ a Instituto de Geociencias (CSIC, UCM), C/ del Doctor Severo Ochoa 7, Ciudad Universitaria, 28040 Madrid, España. jescayo@ucm.es; \\ jt@mat.ucm.es; antonio.camacho@mat.ucm.es \\ b CommSensLab-Universitat Politècnica de Catalunya, D3-Campus Nord-UPC, C/ Jordi Girona 1-3, 08034 Barcelona, España. \\ zhongbo.hu@tsc.upc.edu; mallorqui@tsc.upc.edu \\ ${ }^{\mathrm{c}}$ Canada Centre for Mapping and Earth Observation, Natural Resources Canada, 560 Rochester Street, ON K1A 0E4, Ottawa, Canadá. \\ sergey.samsonov@canada.ca \\ d Escuela Técnica Superior de Ingenieros en Topografía, Geodesia y Cartografía, Universidad Politécnica de Madrid, Campus Sur, A-3, \\ Km 7, 28031 Madrid, España. juanf.prieto@upm.es \\ e Cooperative Institute for Research in Environmental Sciences (CIRES), 216UCB, University of Colorado at Boulder, Boulder, CO, \\ 80309 USA. kristy.tiampo@colorado.edu \\ ${ }^{\mathrm{f}}$ Istituto Nazionale di Geofisica e Vulcanologia, Osservatorio Etneo - Sezione di Catania, Piazza Roma 2, 95125 Catania, Italia. \\ mimmo.palano@ingv.it
}

\begin{abstract}
:
The island of La Palma is one of those with the highest potential risk in the Canary archipelago, therefore it is important to carry out an in-depth study to define its state of volcanic activity. This has been done using satellite radar observations and a state-of-the-art original interpretation technique. Both things have made it possible to detect the beginning of the volcanic reactivation on the island of La Palma, probably decades before a possible eruption. Its temporal evolution shows a changing spatial and temporal nature of volcanic deformation with great spatial resolution and over a prolonged period of time (2009-2020), providing information on the dynamic nature of the associated processes. The geodetic techniques used allow detecting the fluid migration induced by the injection of magma in depth and identifying the existence of dislocation sources under the Cumbre Vieja volcano that could be associated with future landslides, making it necessary to continue with the monitoring of this reactivation process using these and other techniques.
\end{abstract}

Key words: La Palma, geodesy, volcanoes, 3D modelling, remote sensing

\section{Resumen:}

La isla de La Palma es de las de mayor riesgo potencial del archipiélago canario, siendo por tanto importante realizar un estudio en profundidad que permita definir su estado de actividad volcánica. Esto se ha realizado usando observaciones radar de satélite y una técnica de interpretación original de última generación. Ambas cosas han permitido detectar el inicio de la reactivación volcánica en la isla de La Palma, probablemente décadas antes de una posible erupción. Su evolución temporal muestra una naturaleza cambiante de la deformación volcánica, tanto espacial como temporalmente. El uso de imágenes radar permite obtener resultados con una gran resolución espacial y en un periodo de tiempo prolongado (2009-2020), obteniendo información sobre la naturaleza dinámica de los procesos asociados. Las técnicas geodésicas empleadas permiten detectar la migración de fluidos inducida por la inyección de magma en profundidad e identificar la existencia de fuentes de dislocación bajo el volcán Cumbre Vieja que podrían estar asociadas con futuros deslizamientos, siendo por tanto necesario continuar con la monitorización de este proceso de reactivación utilizando estas y otras técnicas.

Palabras clave: La Palma, geodesia, volcanes, modelización 3D, teledetección

\section{Introducción}

Durante las últimas dos décadas se han observado episodios de reactivación volcánica en las Islas Canarias, incluyendo la sismicidad anómala en Tenerife en 20042005, la erupción en El Hierro en 2011-2012 o la reciente sismicidad detectada en La Palma en 2017 y 2018 (Fernández et al. 2015; Martí et al. 2013, 2009). Considerando el riesgo que este tipo de actividad supone

‘Corresponding Author: José Fernández, jft@mat.ucm.es 
a la población y la economía, se hace necesario un estudio en profundidad para definir el estado de reactivación volcánica de las diferentes islas del archipiélago.

Las islas situadas más al oeste, las más jóvenes del archipiélago canario, son El Hierro y La Palma (Fig. 1). La Palma ha estado volcánicamente activa desde hace, por lo menos, $4 \mathrm{Ma}$. Está compuesta por dos unidades volcánicas principales: un gran complejo volcánico en el norte (complejo volcánico norte, NVC) que es la más antigua y posee morfología circular, y un rift volcánico más joven desarrollado en la zona sur de la isla y conocido como complejo de Cumbre Vieja (CV).
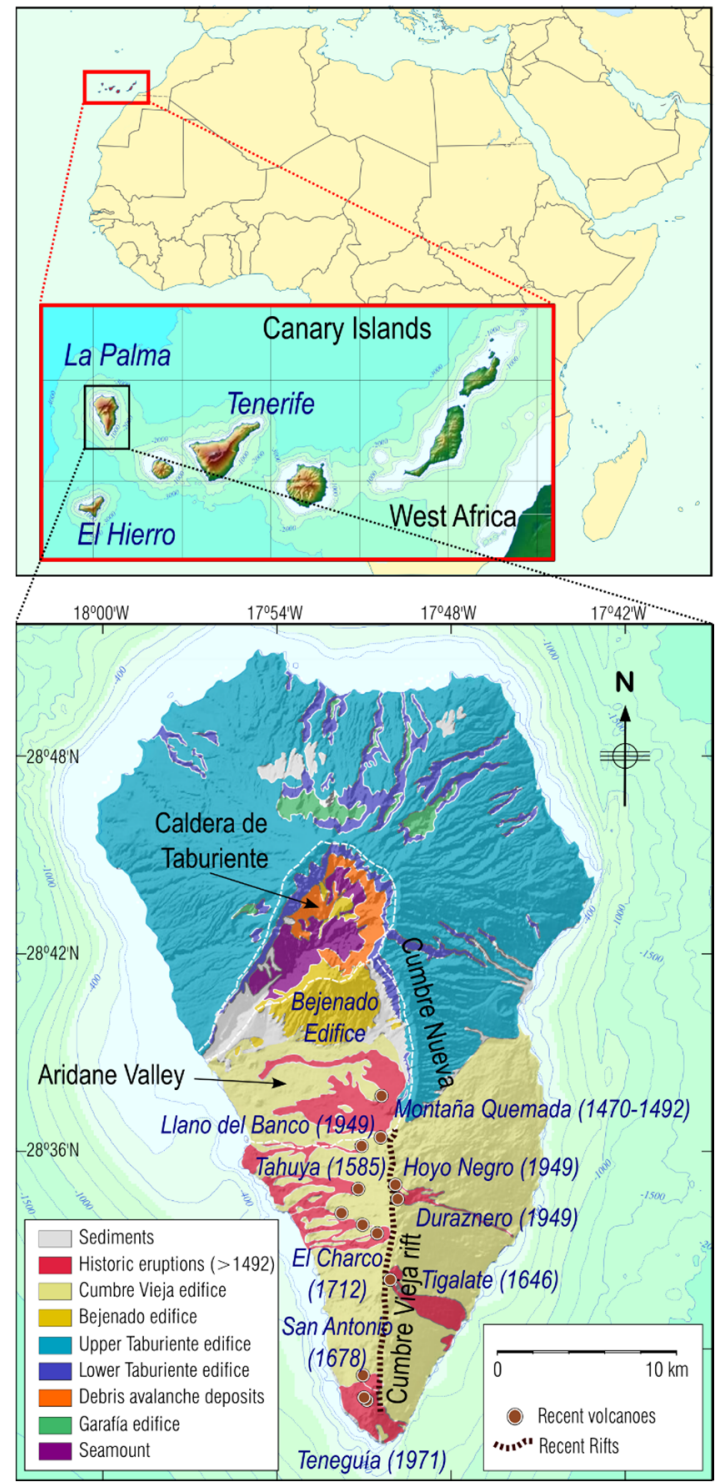

Figura 1: Localización geográfica de La Palma (panel superior) y sus principales elementos geológicos (panel inferior)

(Fernández et al. 2021).

EI NVC es el resultado de la superposición de varios edificios volcánicos basálticos. El edificio más antiguo consiste en cuerpo volcánico submarino cuya edad está comprendida entre 3 y $4 \mathrm{Ma}$ (Staudigel et al. 1986), que tiene superpuestas tres grandes estructuras volcánicas subaéreas que se formaron desde $1.7 \mathrm{Ma}$ a $0.4 \mathrm{Ma}$ (Ancochea et al. 1994; Carracedo et al. 2001): el edificio de Garafía (1.7 a 1.2 Ma), el eficio de Taburiente-Cumbre
Nueva, T-CN (1.2-0.4 Ma), y el edificio de Bejenado (0.56-0.49 Ma) (Fig. 1). NVC alcanza los $2426 \mathrm{~m}$ de altura. Grandes deslizamientos de ladera y fenómenos erosivos han afectado a NVC, exponiendo parte de la estructura submarina, cuerpos plutónicos y diques que constituyen las raíces de los edificios en el fondo de la Caldera de Taburiente. La parte submarina de la isla está inclinada y elevada unos $300 \mathrm{~m}$ de altitud, bajo los edificios de Garafía y Taburiente y ahora se pueden ver en la base de la caldera de Taburiente. El edicidio T-CN, que cubre los más antiguos, en su primera fase morfológica (1.2-0.8 Ma) tiene forma circular, lo que hace que la isla adopte esta forma en la zona norte. Sin embargo, en su segunda fase (0.8-0.4 Ma) su morfología es aproximadamente igual a la del rift $\mathrm{N}-\mathrm{S}$ de $\mathrm{CN}$.

La segunda mayor estructura volcánica de la isla es el edificio CV cuya actividad comenzó posteriormente a los edificios anteriores. El rift tiene una orientación N-S, como en el caso de $\mathrm{CN}$, con una altitud máxima $1950 \mathrm{~m}$ y una longitud subáerea de aproximadamente $17 \mathrm{~km}$ que se extiende varios kilómetros en el fondo marino (Urgeles et al. 1999). La actividad volcánica más reciente en la isla se concentra en este edificio volcánico, incluyendo las seis erupciones históricas riencientes que han tenido lugar en los últimos 500 años. Uno de los últimos eventos fue la erupción de San Juan en 1949 cuando se registró actividad eruptiva en tres conos volcánicos: Duraznero, Llano del Blanco y Hoyo Negro (Fig. 1). La última erupción sub-aérea en La Palma (y en las Islas Canarias) fue en 1971 y ocurrió en el volcán Teneguía, hace aproximadamente 50 años (Fig. 1). Las reactivaciones volcánicas en La Palma, y especialmente en el área de $\mathrm{CV}$, también se han asociado con deslizamientos de ladera y riesgos de tsunamis (Ward and Day 2001). Torres-González et al. (2020) encontraron evidencias de una intrusión magmática localizada a unos $25 \mathrm{~km}$ de profundidad en La Palma, basándose en el análisis de la sismicidad y anomalías geoquímicas durante el periodo de 2017-2018, aunque no detectaron deformación superficial con GNSS contínuo. Esto, junto con los riesgos asociados a una posible reactivación volcánica, motivaron el estudio de la deformación superficial utilizando para ello técnicas de interferometría radar de apertura sintética (DInSAR).

Históricamente, la sismicidad ha sido el primer precursor detectado en procesos de reactivación volcánica en las Canarias, mientras que la deformación superficial detectada durante episodios de reactivación no ha sido importante (Fernández et al. 2015, 2021). En El Hierro, en 2011, se detectaron deformaciones del orden de la decena de centímetros unos meses antes de la erupción. En Tenerife, durante la activación de 2004-2005, la deformación observada fue del orden de $2-3 \mathrm{~cm}$, con una baja relación señal/ruido (Fernández et al. 2015). Obtener deformaciones a partir de técnicas DInSAR es complicado en entornos como La Palma, ya que la existencia de grandes desniveles hace que los efectos atmosféricos tengan especial importancia, requiriendo el uso de técnicas avanzadas para poder corregir su contribución de los valores de deformación. El uso de datos GNSS se ve limitado por la dificultad de realizar campañas con un gran número de puntos de observación (Escayo et al. 2020; Fernandez et al. 2018), así como la pequeña magnitud de los desplazamientos (Fernández et al. 2017). En La Palma, como también ocurre en otras muchas islas volcánicas, la localización de las 
deformaciones asociadas a posibles reactivaciones no son conocidas a priori y pueden variar con el tiempo. Esto hace que las redes GNSS con una baja densidad espacial puedan resultar de poca utilidad para la detección de deformaciones de pequeña magnitud, principalmente en las etapas iniciales de una reactivación (Escayo et al. 2020; Torres-González et al. 2020).

Para solventar estas limitaciones se emplean las técnicas DInSAR, ya que permiten la obtención de mapas de deformación con una gran resolución espacial (tamaños de píxel habituales de $1 \times 1 \mathrm{~m}$ para banda $X$ y $5 \times 20$ para banda C) y con alta precisión (los análisis de series temporales son capaces de determinar velocidades medias anuales con precisiones milimétricas). Además, mediante el uso de técnicas avanzadas, p.e. modelos climáticos globales como el caso de ERA5, podemos realizar un correcto filtrado atmosférico. Todo esto, combinado con un modelo de inversión avanzado (Camacho et al. 2020), que es capaz de hacer una inversión considerando múltiples fuentes de deformación, obteniendo la localización, tamaño y configuración tridimensional sin hacer ninguna hipótesis a priori del número, naturaleza o forma de la fuente. Adicionalmente, los resultados se discutirán considerando la estructura cortical tridimensional de la isla determinada a partir de observaciones gravimétricas.

\section{Resultados}

\subsection{DInSAR}

El estudio DInSAR se realizó utilizando imágenes Single Look Complex (SLC) adquiridas por diferentes satélites de banda $C$. Se consideran tres periodos de tiempo: 2006- 2010 donde se utilizó el satélite ENVISAT; 20102017 utilizando imágenes de satélite RADARSAT-2; y 2017-2020 donde se utilizó la constelación de satélites Sentinel-1. Los satélites ENVISAT y la constelación Sentinel-1 fueron puestos en órbita por la Agencia Espacial Europea (ESA) mientras que el satélite RADARSAT-2 fue puesto en órbita por la Agencia Espacial Canadiense (CSA). Todos los datos utilizados en este trabajo fueron suministrados por las respectivas agencias espaciales. Para eliminar la contribución a la fase debida por la topografía se utilizó un modelo digital del terreno de alta resolución generado a partir de los datos del MDT05 proporcionados por el Instituto Geográfico Nacional (IGN) descargados desde el centro de descargas del CNIG.

Para obtener los desplazamientos en la línea de visión (o Line of Sight, LOS) del satélite se utilizó la técnica "Coherent Pixel Technique" (CPT) (Blanco-Sánchez et al. 2008) para los conjuntos de datos de ENVISAT y Sentinel-1. Se aplicó una selección de píxeles por coherencia (técnica similar al SBAS) aplicando un multilook sobre la imagen de 5x25 muestras (obteniendo así una resolución cercana a los 100×100 m). Para seleccionar los píxeles con suficiente calidad de fase se aplicó un umbral de coherencia de 0.4 sobre la coherencia media generada a partir de todos los interferogramas. Para eliminar los efectos atmosféricos (Atmospheric Phase Screen) presentes en la isla se utilizaron dos técnicas diferentes dependiendo del sensor: para ENVISAT se utilizó un filtrado espaciotemporal mientras que pasa Sentinel-1 se generaron mapas de APS sintéticos a partir de los datos de los modelos globales de ERA5 (Hu and Mallorquí 2019), permitiendo una estimación más precisa de los efectos atmosféricos.

Para el conjunto de datos de RADARSAT-2 se utilizó el software MSBAS (Samsonov and D'Oreye 2017) con una ventana de multilook de $4 \times 8$ muestras, permitiendo una resolución de aproximadamente $40 \times 40 \mathrm{~m}$. Para la estimación del APS se calculó una correlación entre topografía y efecto atmosférico, usando el modelo digital del terreno.

En este trabajo se intentó disponer de la mayor cantidad de datos posible para el procesado, obteniendo órbitas ascendentes y descendentes para los satélites ENVISAT y la constelación Sentinel-1, mientras que para el satélite RADARSAT-2 únicamente se disponía de suficientes imágenes para el análisis DInSAR en la órbita descendente. Para todos los casos se calcularon las velocidades medias en LOS (Fig. 2), y en el caso de ENVISAT y Sentinel-1 se obtuvieron también las series temporales para cada uno de los tres periodos estudiados. Con el fin de validar los resultados obtenidos se compararon los valores de diferentes puntos GNSS de los que se disponían datos con los píxeles más cercanos obtenidos mediante DInSAR mostrando diferencias para las velocidades medias anuales inferiores a $4 \mathrm{~mm} / \mathrm{y}$ en el caso de ENVISAT y $2 \mathrm{~mm} / \mathrm{y}$ para Sentinel-1, lo que consideramos es una buena concordancia entre ambas técnicas. Las velocidades medias de RADARSAT-2 no se utilizaron para su inversión puesto que únicamente se disponía de una geometría de adquisición, lo que resultaba insuficiente para obtener una buena inversión.
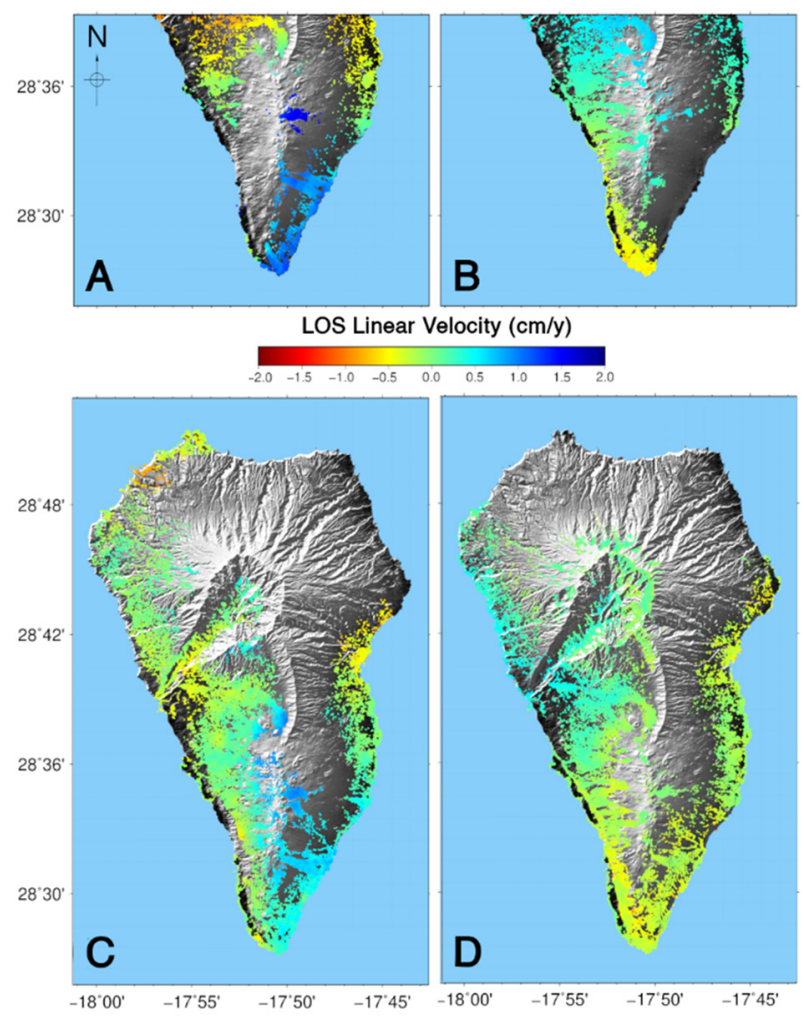

Figura 2: Velocidades medias anuales para los diferentes periodos estudiados: A) Periodo 2006-2010 en órbita ascendente; B) Periodo 2006-2010 en órbita descendente; C) Periodo 2017-2020 en órbita ascendente; D) Periodo 20172020 en órbita descendente. Modificado de Fernández et al. (2021). 


\subsection{Inversión}

Los datos de velocidades medias de deformación para los tres periodos fueron modelados para estimar la naturaleza, localización y geometría de las fuentes causantes utilizando la metodología de inversión de Camacho et al. (2020). El resultado del ajuste libre de los datos disponibles en LOS, para cada periodo considerado, permite obtener las fuentes de deformación como conjuntos de celdas 3D para los cuales el software de inversión ajusta automáticamente y asigna su tipo (cambios de presión, o diferentes tipos de dislocaciones: desgarre/direccional, deslizamiento y extensional/ compresional), su magnitud (MPa para cambios de presión y centímetros para dislocaciones) y su posición y orientación (ángulos de los planos de dislocación).

Las fuentes modeladas se han representado en la Figura 3 para los periodos 2006-2010 y 2017-2020, coloreadas según su naturaleza. Dado que la cobertura espacial de los resultados DInSAR se ve afectada por la decorrelación temporal, para asegurar que el conjunto de datos disponía de suficiente densidad espacial para ser invertido se calcularon los valores LOS de las órbitas ascendentes y descendentes para las fuentes modeladas utilizando los modelos para cada tipo de fuente (Camacho et al. 2020) y se compararon los valores obtenidos con los observados. El ajuste entre ambos resultados es bastante bueno para ambas geometrías y los residuos (diferencia entre observado-modelado) tienen valores RMS inferiores a $0.4 \mathrm{~cm} / \mathrm{y}$.

En el caso del periodo 2010-2017 (datos del satélite RADARSAT-2) únicamente se dispone de velocidades medias para la órbita descendente y en su cálculo se ha utilizado un método para estimación del APS y su posterior filtrado que no es lo suficientemente preciso para un caso como La Palma, donde la topografía y ubicación hacen que presente un fuerte efecto atmosférico difícilmente modelable únicamente a partir de datos de elevación. Uno de los efectos que contribuyen a esto es el viento, que debido a que tiene una dirección predominante hace que la humedad se concentre en una de las costas de la isla. Los resultados de la inversión se muestran fuertemente afectados por estos aspectos y por ello no los hemos incluido en nuestro estudio.

Para el periodo 2017-2020 hemos sido capaces de estudiar la evolución temporal de las fuentes modeladas. La evolución temporal de las fuentes positivas de presión para el periodo 2017-2020 se muestra en la Figura 4. Este tipo de análisis no ha sido posible para el periodo 2006-2010 debido al bajo número de imágenes y la pobre distribución temporal de que dispone el satélite ENVISAT.

\subsection{Gravimetría estructural}

Es necesario disponer de cierta información básica, como por ejemplo la estructura cortical de la isla, para poder discutir los resultados de la inversión del campo de deformación. Utilizaremos la distribución de densidades $3 \mathrm{D}$ por debajo de la superficie de la isla obtenido a partir de mediciones gravimétricas.

Utilizando datos de campañas gravimétricas que cubren la superficie de la isla (Camacho et al. 2009; Prieto et al. 2009), y la metodología descrita en Camacho et al. (2011) hemos obtenido un modelo tridimensional para las anomalías en la distribución de densidad para la corteza de la isla. Los resultados se muestran en la Figura 5.
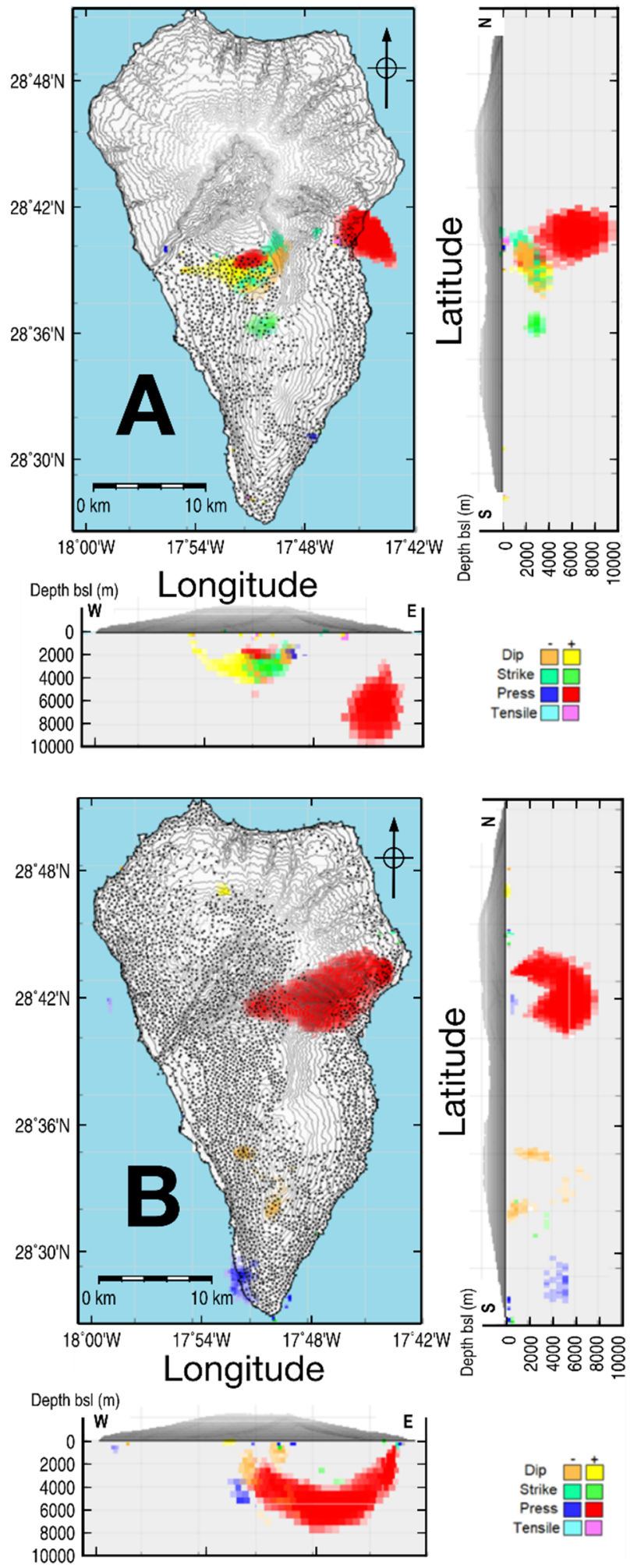

Figura 3: Ajuste del modelo para las fuentes de presión: A) Fuentes para el periodo 2006-2010; B) Fuentes para el periodo 2017-2020. En ambos casos se incluyen vistas zenital, E-W vertical y N-S vertical de las fuentes significativas. En la escala se representan los tipos de fuente y su magnitud. Modificado de Fernández et al. (2021). 


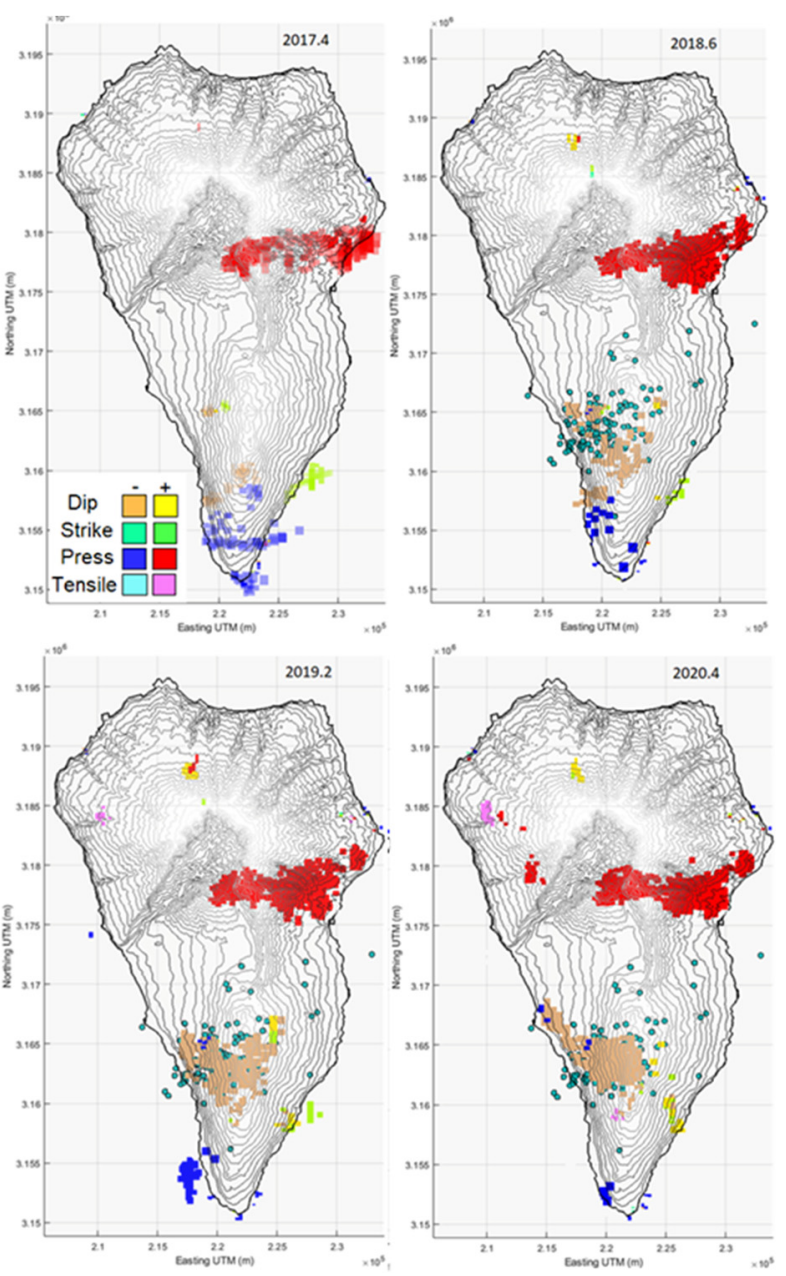

Figura 4: Evolución temporal de las fuentes obtenidas a partir del modelo para el periodo 2017-2020. Se han obtenido a partir de las series temporales en ambas geometrías (ascendente y descendente) y representado para diferentes fechas del periodo 2017-2020. Los círculos verdes representan la localización de los terremotos ocurridos durante los dos ejambres sísmicos registrados (octubre 2017 y febrero 2018). Modificado de Fernández et al. (2021).

La característica más destacable del modelo de inversión es un gran cuerpo central de gran densidad localizado debajo de NVC (Fig. 1) y que representa la existencia de un denso cuerpo intrusivo que contrasta con el material de baja densidad que lo rodea (material eruptivo no compactado con alto grado de vesiculación, fracturas o poros). Esta es la zona donde los diques radiales que alimentan el complejo del cuerpo volcánico submarino de Garafía y el eficio de T-CN convergen (Ancochea et al. 1994) y se localiza el cuerpo plutónico relativamente denso que no llegó a alcanzar la superficie.

La mitad sur de la isla, que principalmente consiste en el edificio CV (125 ka hasta la actualidad), esta caracterizado por mínimos distribuidos por las principales estructuras del rift. Se detectan mínimos significativos a profundidades de 1000-2000 m bajo del nivel del mar por debajo de la pendiente oeste del CV y distribuidas de manera escalonada con un azimuth de $\mathrm{N} 130^{\circ} \mathrm{E}$. Esto se interpreta como asociado a proceso activo y lento de dislocación (Camacho et al. 2009).

\section{Discusión y conclusiones}

A partir de los resultados, podemos obtener varias hipótesis y conclusiones acerca de las fuentes responsables de las deformaciones observadas. En el caso de las fuentes de presión, debemos considerar la posibilidad de que no estén provocadas por magma y sean producidas por otras causas. Las fuentes de presión positivas, de baja intensidad, son importantes debido a sus implicaciones en el estado de actividad volcánica en la isla (Fernández et al. 2021).

Un aspecto importante es la detección de deformación en el Valle de Aridane (Fig. 2) mientras que en el estudio previo de González et al. (2010) no fue detectada durante los periodos $1992-2000$ y 2003-2008. Esto, junto a otros resultados previos que no muestran anomalías antes de 2009 , parece indicar que la fuente causante de la deformación superficial probablemente se activó entre 2009 y 2010, indicando el comienzo de una reactivación volcánica en la isla de La Palma tras cerca de 40 años de inactividad.

Se ha detectado un incremento en la componente magmática en las emisiones de helio en la estación de Dos Aguas en 2010, así como emisiones anómalas de CO2 en las campañas llevadas a cabo en CV en 2011 y 2013 (Padrón et al. 2015). Estas variaciones es muy probable que hayan ocurrido como respuesta a eventos que sucediesen cerca de un año antes (Padrón et al. 2015) y apoyan la hipótesis de que se produjo el inicio de una fase de reactivación.

La deformación producida por intrusiones superficiales suelen ocurrir durante periodos de reactivación y es habitual que se produzcan terremotos con intensidades que pueden ser sentidos por la población (González et al. 2010). Este no ha sido el caso para el periodo 2006-2010, donde muy pocos terremotos tuvieron lugar en la zona de estudio. Sin embargo, las reactivaciones pueden tener una sismicidad muy baja o indetectable (McNutt and Roman 2015). Por ejemplo, si consideramos la actividad volcánica reciente en la zona, la sismicidad precursora a la erupción de 1949 (Klügel et al. 2000) comenzó en 1936 en el Valle de Aridane, a $5-10 \mathrm{Km}$ de los conos volcánicos, y continuaron durante unos meses antes de la erupción en la zona de Fuencaliente, a unos $10 \mathrm{~km}$ del cono de Duraznero. Los conos de Llano del Blanco y Hoyo Negro se abrieron sin que existiese sismicidad precursora. Entre 1936 y 1949 el magma ascendió desde el manto superior y se acumuló a diferentes niveles en la corteza sin que se sintiese actividad sísmica.

La actividad sísmica reciente empezó con dos enjambres sísmicos en 2017 y 2018 localizados a aproximadamente $15 \mathrm{~km}$ al sur de las fuentes de presión modeladas (Torres-González et al. 2020). Por tanto, podemos estar estudiando una fase muy inicial de reactivación volcánica decenas de años antes de una posible erupción (Fisher and Schmincke 1984), si esta llegase a ocurrir. Se estima que alrededor del $80 \%$ de los magmas que se forman en el entorno oceánico intraplaca no entran en erupción (Fernández et al. 2021).

Al observar la variación temporal de las fuentes modeladas en 2017-2020 (Fig. 4), se observa que la presión positiva de la fuente aumenta hasta 2019, casi con certeza relacionada con los dos enjambres sísmicos, que probablemente abrieron nuevas fracturas facilitando 
el ascenso de gases volcánicos y magma (Fernández et al. 2021). El mayor incremento parece estar asociado con los dos enjambres sísmicos entre 2017 y 2018. Ambos enjambres sísmicos fueron precedidos y acompañados de cambios en las emisiones de helio y dióxido de carbono, lo que sugiere una intrusión magmática por debajo de $\mathrm{CV}$.

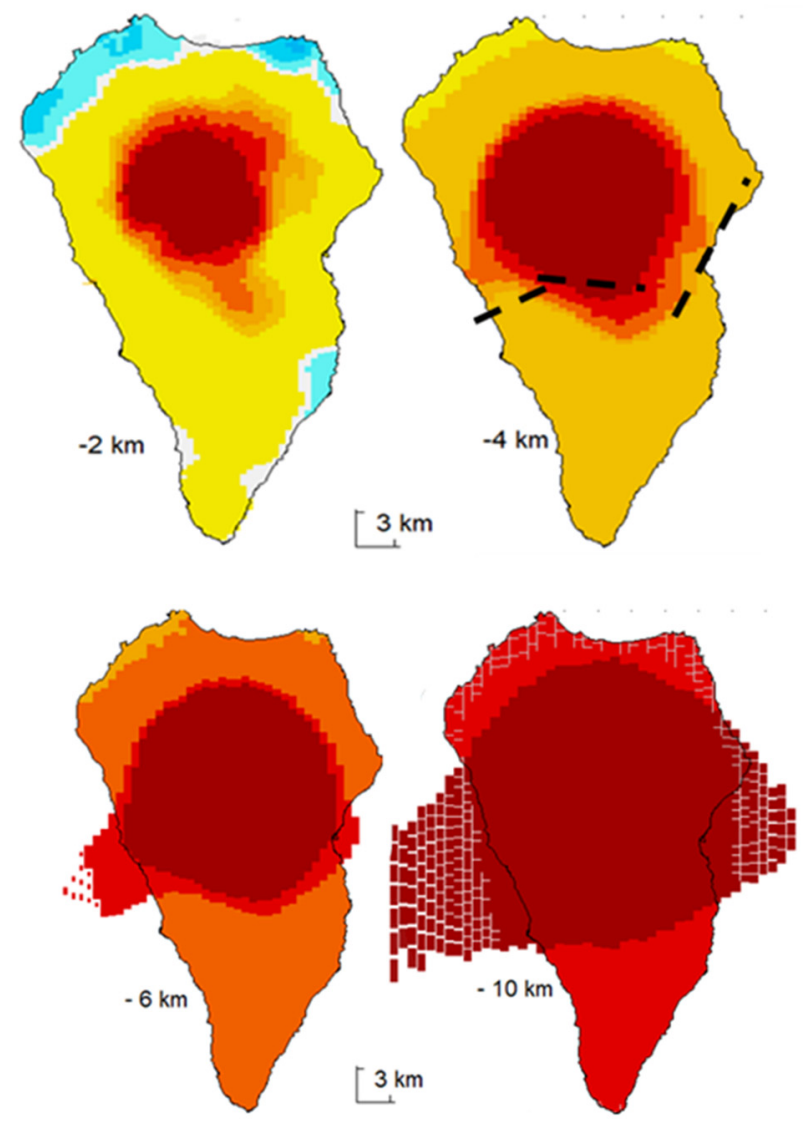

Figura 5: Secciones horizontales de la estructura anómala de densidad tridimensional y localización de las fuentes de presión modeladas para el periodo 2006-2010. Las secciones horizontales están tomadas a diferentes profundidades desde 2 $\mathrm{km}$ hasta $10 \mathrm{~km}$ bajo el nivel del mar. Modificado de Fernández et al. (2021)

La presión siguió aumentando durante 2019, probablemente asociada a una recarga asísmica. A partir de 2019, la presión promedio permanece constante o disminuye. Las fuentes de dislocación bajo CV son poco intensas en el período 2017-2018, pero a partir de 2019 aumentan, contrariamente a los valores de presión. Su ubicación (Fig. 4) coincide con el área donde la mayoría de los terremotos de los enjambres sísmicos se localizan y comenzaron a crecer varios meses después del segundo enjambre (Fernández et al. 2021). Estas fuentes podrían estar asociadas con fracturas y, considerando el peligro de deslizamiento de flanco existente, su evolución necesita seguir siendo monitorizada e interpretada.

El uso de técnicas DInSAR (con una corrección atmosférica en el estado del arte y utilizando ambas geometrías de adquisición, ascendente y descendente) resulta ser una herramienta fundamental para la monitorización y la detección de una posible reactivación volcánica teniendo en cuenta la distribución espacial y temporal de la deformación observada. Realizar campañas de medición GNSS puede resultar muy difícil y costoso en un entorno como La Palma, además de poder encontrar situaciones donde la magnitud de la deformación se encuentra en el límite de precisión ofrecido por este tipo de mediciones haciendo que no sean detectados este tipo de deformaciones (Escayo et al. 2020; Fernández et al. 2018, 2021). Sin embargo, disponer de estaciones GNSS resulta crucial para poder establecer puntos de referencia para las técnicas DInSAR (Fernández et al. 2018, 2021).

Los resultados obtenidos a partir de la inversión conjunta de los conjuntos de datos ascendente y descendente presentan una variedad de fuentes actuando simultáneamente, lo que no sería posible obtener a partir de técnicas de inversión y modelos clásicos que únicamente consideran fuentes magmáticas. Esto es importante en La Palma, donde se pueden producir deslizamientos de ladera como consecuencia de activad volcánica.

\section{Agradecimientos}

Esta investigación ha sido financiada por el proyecto de investigación DEEP-MAPS (RTI2018-093874-B-100) del Ministerio de Ciencia, Innovación y Universidades. También ha sido parcialmente financiado por el proyecto del CSIC 201530E019, el proyecto GEOSIR (AYA201017448) del Ministerio de Ciencia e Innovación y el proyecto del MINECO CGL2017-86241-R. Agradecemos a DARES S.L por la licencia de uso del software interferométrico CPT en este studio. Este trabajo representa una contribución a la plataforma interdisciplinar del CSIC PTI TELEDETECT.

\section{References}

ANCOCHEA, E., HERNÁN, F., CENDRERO, A., CANTAGREL, J.M., FÚSTER, J., IBARROLA, E., and COELLO, J., 1994. Constructive and destructive episodes in the building of a young Oceanic Island, La Palma, Canary Islands, and genesis of the Caldera de Taburiente. J. Volcanol. Geotherm. Res., 60, pp. 243-262. DOI: 10.1016/0377-0273(94)90054-X

BLANCO-SÁNCHEZ, P., MALLORQUÍ, J.J., DUQUE, S., and MONELLS, D., 2008. The coherent pixels technique (CPT): An advanced DInSAR technique for nonlinear deformation monitoring. Pure Appl. Geophys., 165, pp. 1167-1193. DOI: 10.1007/s00024-008-0352-6

CAMACHO, A.G., FERNÁNDEZ, J., GONZÁLEZ, P.J., RUNDLE, J.B., PRIETO, J.F., and ARJONA, A., 2009. Structural results for La Palma island using 3-D gravity inversion. J. Geophys. Res., 114, B05411. DOI: 10.1029/2008JB005628

CAMACHO, A.G., FERNÁNDEZ, J., and GOTTSMANN, J., 2011. A new gravity inversion method for multiple subhorizontal discontinuity interfaces and shallow basins. J. Geophys. Res. Solid Earth, 116. DOI: 10.1029/2010JB008023 
CAMACHO, A.G., FERNÁNDEZ, J., SAMSONOV, S. V., TIAMPO, K.F., and PALANO, M., 2020. 3D multi-source model of elastic volcanic ground deformation. Earth Planet. Sci. Lett., 547, 116445. DOI: 10.1016/j.epsl.2020.116445

CARRACEDO, J.C., BADIOLA, E.R., GUILLOU, H., DE LA NUEZ, J., and PÉREZ TORRADO, F.J., 2001. Geology and volcanology of La Palma and El Hierro, Western Canaries. Estud. Geol., 57, pp. 175-273. DOI: 10.3989/egeol.015756134

ESCAYO, J., FERNÁNDEZ, J., PRIETO, J.F., CAMACHO, A.G., PALANO, M., APARICIO, A., RODRÍGUEZ-VELASCO, G., and ANCOCHEA, E., 2020. Geodetic Study of the 2006-2010 Ground Deformation in La Palma (Canary Islands): Observational Results. Remote Sens., 12, 2566. DOI: 10.3390/rs12162566

FERNÁNDEZ, J., ESCAYO, J., HU, Z., CAMACHO, A. G., SAMSONOV, S. V., PRIETO, J. F., TIAMPO,K.F., PALANO, M., MALLORQUÍ, J.J., and ANCOCHEA, E., 2021. Detection of volcanic unrest onset in La Palma, Canary Islands, evolution and implications. Scientific reports, 11(1), pp. 1-15. DOI: 10.1038/s41598-021-82292-3

FERNÁNDEZ, J., GONZÁLEZ, P.J., CAMACHO, A.G., PRIETO, J.F., and BRÚ, G., 2015. An Overview of Geodetic Volcano Research in the Canary Islands. Pure Appl. Geophys., 172, pp. 3189-3228. DOI: 10.1007/s00024-014-09166

FERNÁNDEZ, J., PEPE, A., POLAND, M.P., and SIGMUNDSSON, F., 2017. Volcano Geodesy: Recent developments and future challenges. J. Volcanol. Geotherm. Res., 344, pp. 1-12. DOI: 10.1016/j.jvolgeores.2017.08.006

FERNÁNDEZ, J., PRIETO, J.F., ESCAYO, J., CAMACHO, A.G., LUZÓN, F., TIAMPO, K.F., PALANO, M., ABAJO, T., PÉREZ, E., VELASCO, J., HERRERO, T., BRU, G., MOLINA, I., LÓPEZ, J., RODRÍGUEZ-VELASCO, G., GÓMEZ, I., and MALLORQUÍ, J.J., 2018. Modeling the two- and three-dimensional displacement field in Lorca, Spain, subsidence and the global implications. Sci. Rep., 8, 14782. DOI: 10.1038/s41598-018-33128-0

FISHER, R. V., and SCHMINCKE, H.U., 1984. Pyroclastic rocks. Pyroclastic rocks. DOI: 10.1029/eo066i009p00092-03

GONZÁLEZ, P.J., TIAMPO, K.F., CAMACHO, A.G., and FERNÁNDEZ, J., 2010. Shallow flank deformation at Cumbre Vieja volcano (Canary Islands): Implications on the stability of steep-sided volcano flanks at oceanic islands. Earth Planet. Sci. Lett., 297, pp. 545-557. DOI: 10.1016/j.epsl.2010.07.006

HU, Z., and MALLORQUÍ, J.J., 2019. An Accurate Method to Correct Atmospheric Phase Delay for InSAR with the ERA5 Global Atmospheric Model. Remote Sens., 11, 1969. DOI: 10.3390/rs11171969

KLÜGEL, A., HOERNLE, K.A., SCHMINCKE, H.-U., and WHITE, J.D.L., 2000. The chemically zoned 1949 eruption on La Palma (Canary Islands): Petrologic evolution and magma supply dynamics of a rift zone eruption. J. Geophys. Res. Solid Earth, 105, pp. 5997-6016. DOI: 10.1029/1999JB900334

MARTÍ, J., ORTIZ, R., GOTTSMANN, J., GARCIA, A., and DE LA CRUZ-REYNA, S., 2009. Characterising unrest during the reawakening of the central volcanic complex on Tenerife, Canary Islands, 2004-2005, and implications for assessing hazards and risk mitigation. J. Volcanol. Geotherm. Res., 182, pp. 23-33. DOI: 10.1016/j.jvolgeores.2009.01.028

MARTí, J., PINEL, V., LÓPEZ, C., GEYER, A., ABELLA, R., TÁRRAGA, M., BLANCO, M.J., CASTRO, A., and RODRÍGUEZ, C., 2013. Causes and mechanisms of the 2011-2012 El Hierro (Canary Islands) submarine eruption. J. Geophys. Res. Solid Earth, 118, pp. 823-839. DOI: 10.1002/jgrb.50087

MCNUTT, R., and ROMAN, D.C., 2015. Volcanic Seismicity. The Encyclopedia of Volcanoes. pp. 1011-1034. DOI: 10.1016/B978-0-12-385938-9.00059-6

PADRÓN, E., PÉREZ, N.M., RODRÍGUEZ, F., MELIÁN, G., HERNÁNDEZ, P.A., SUMINO, H., PADILLA, G., BARRANCOS, J., DIONIS, S., NOTSU, K., and CALVO, D., 2015. Dynamics of diffuse carbon dioxide emissions from Cumbre Vieja volcano, La Palma, Canary Islands. Bull. Volcanol., 77, p. 28. DOI: 10.1007/s00445-015-0914-2

PRIETO, J.F., GONZÁLEZ, P.J., SECO, A., RODRÍGUEZ-VELASCO, G., TUNINI, L., PERLOCK, P.A., ARJONA, A., APARICIO, A., CAMACHO, A.G., RUNDLE, J.B., TIAMPO, K.F., PALLERO, J.L.G., POSPIECH, S., and FERNÁNDEZ, J., 2009. Geodetic and Structural Research in La Palma, Canary Islands, Spain: 1992-2007 Results. Pure Appl. Geophys., 166, pp. 1461-1484. DOI: 10.1007/s00024-009-0505-2

SAMSONOV, S. V., and D'OREYE, N., 2017. Multidimensional Small Baseline Subset (MSBAS) for Two-Dimensional Deformation Analysis: Case Study Mexico City. Can. J. Remote Sens., 43, pp. 318-329. DOI: 10.1080/07038992.2017.1344926

STAUDIGEL, H., FERAUD, G., and GIANNERINI, G., 1986. The history of intrusive activity on the island of La Palma (Canary Islands). J. Volcanol. Geotherm. Res., 27, pp. 299-322. DOI: 10.1016/0377-0273(86)90018-1

TORRES-GONZÁLEZ, P.A., LUENGO-OROZ, N., LAMOLDA, H., D’ALESSANDRO, W., ALBERT, H., IRIBARREN, I., MOURE-GARCÍA, D., and SOLER, V., 2020. Unrest signals after 46 years of quiescence at Cumbre Vieja, La Palma, Canary Islands. J. Volcanol. Geotherm. Res., 392, 106757. DOI: 10.1016/j.jvolgeores.2019.106757

URGELES, R., MASSON, D.G., CANALS, M., WATTS, A.B., and LE BAS, T., 1999. Recurrent large-scale landsliding on the west flank of La Palma, Canary Islands. J. Geophys. Res. Solid Earth, 104, pp. 25331-25348. DOI: 10.1029/1999JB900243 
WARD, S. N., and DAY, S., 2001. Cumbre Vieja volcano-potential collapse and tsunami at La Palma, Canary Islands. Geophysical Research Letters, 28(17), pp. 3397-3400. DOI: 10.1029/2001GL013110 